\title{
Moldagens em prótese sobre implantes / moldeira fechada ou aberta?
}

\author{
Prosthesis molding on implants / closed or open tray? \\ Prótesis moldura en implantes / bandeja cerrada o abierta?
}

Recebido: 26/07/2021 | Revisado: 30/07/2021 | Aceito: 05/08/2021 | Publicado: 10/08/2021

\author{
Patrícia Nunes da Rocha \\ ORCID: https://orcid.org/0000-0002-1510-1985 \\ Faculdade Patos de Minas, Brasil \\ E-mail: patyrocha89@hotmail.com \\ Marcelo Dias Moreira de Assis Costa \\ ORCID: https://orcid.org/0000-0001-9148-3674 \\ Universidade Federal de Uberlândia, Brasil \\ E-mail: marcelodmac@yahoo.com.br \\ Lia Dietrich \\ ORCID: https://orcid.org/0000-0001-7887-8591 \\ Universidade Federal do Vale do Jequitinhonha e Mucuri, Brasil \\ E-mail: lia_dietrich@yahoo.com.br
}

\begin{abstract}
Resumo
Como objetivo principal deste estudo tem-se: ampliar os conhecimentos sobre as técnicas de moldagem em moldeiras abertas e fechadas. Como objetivos secundários, propõe-se: estudar sobre as próteses sobre implantes; caracterizar as distinções entre as moldeiras abertas e fechadas; ampliar os conhecimentos sobre as situações mais adequadas para o uso de cada estilo de moldeira (aberta ou fechada). O presente estudo trata-se de um levantamento bibliográfico descritivo e qualitativo, utilizando-se assim de referenciais teóricos extraídos de livros, artigos científicos, monografias, teses, dissertações revistas e sites eletrônicos que retratem o tema proposto. Nos últimos anos, a demanda para a reabilitação de perdas dentárias com implantes, se popularizou. No atual estágio de desenvolvimento que as próteses sobre implantes atingiram, estas são consideradas como sendo as melhores opções de tratamento, sendo consideradas como de alto padrão dentro das próteses dentarias. O principal objetivo de uma moldagem em prótese sobre implantes é registrar com exatidão o posicionamento dos componentes na boca e dos tecidos moles, bem como das estruturas adjacentes ao espaço edêntulo, utilizando-se assim componentes específicos para transferência, bem como análogos metálicos do dispositivo presente em boca, seja pilares ou implantes. Entre as técnicas de moldagens temos a técnica com moldeira aberta e com moldeira fechada. Portanto, a escolha entre moldeira aberta ou fechada, será baseada nas características dos componentes e reabilitação que serão realizados. Ambas as técnicas possuem suas vantagens, no entanto, cada uma é indicada para casos específicos. Neste sentido, a escolha se baseará na demanda individual de cada paciente.
\end{abstract}

Palavras-chave: Implantes dentários; Moldeira aberta; Moldeira fechada; Prótese dentária.

\begin{abstract}
The main objective of this study is: to increase knowledge about molding techniques in open and closed trays. As secondary objectives, it is proposed: to study about implant prostheses; characterize the distinctions between open and closed trays; expand knowledge about the most suitable situations for the use of each tray style (open or closed). The present study is a descriptive and qualitative bibliographic survey, using theoretical references extracted from books, scientific articles, monographs, theses, revised dissertations and electronic websites that portray the proposed theme. In recent years, the demand for the rehabilitation of tooth loss with implants has become popular. In the current stage of development that implant prostheses have reached, they are considered to be the best treatment options, being considered as a high standard within dental prostheses. The main objective of an implant prosthesis molding is to accurately record the positioning of the components in the mouth and soft tissues, as well as the structures adjacent to the edentulous space, thus using specific components for transfer, as well as metallic analogues of the present device. in the mouth, whether abutments or implants. Among the molding techniques we have the technique with open tray and with closed tray. Therefore, the choice between open or closed tray will be based on the characteristics of the components and rehabilitation that will be carried out. Both techniques have their advantages, however, each is indicated for specific cases. In this sense, the choice will be based on the individual demand of each patient.
\end{abstract}

Keywords: Dental implants; Open tray; Closed tray; Prothesis.

\section{Resumen}

El objetivo principal de este estudio es: incrementar el conocimiento sobre las técnicas de moldeo en bandejas abiertas y cerradas. Como objetivos secundarios, se propone: estudiar sobre prótesis sobre implantes; caracterizar las 
distinciones entre bandejas abiertas y cerradas; ampliar el conocimiento sobre las situaciones más adecuadas para el uso de cada estilo de bandeja (abierta o cerrada). El presente estudio es un levantamiento bibliográfico descriptivo y cualitativo, utilizando referencias teóricas extraídas de libros, artículos científicos, monografías, tesis, disertaciones revisadas y sitios web electrónicos que retratan la temática propuesta. En los últimos años, se ha popularizado la demanda de rehabilitación de la pérdida de dientes con implantes. En la actual etapa de desarrollo que han alcanzado las prótesis sobre implantes, se consideran las mejores opciones de tratamiento, considerándose como un alto estándar dentro de las prótesis dentales. El principal objetivo del moldeado de una prótesis de implante es registrar con precisión el posicionamiento de los componentes en la boca y tejidos blandos, así como las estructuras adyacentes al espacio edéntulo, utilizando así componentes específicos para la transferencia, así como análogos metálicos del presente. dispositivo en la boca, ya sean pilares o implantes. Entre las técnicas de moldeo tenemos la técnica con bandeja abierta y con bandeja cerrada. Por tanto, la elección entre bandeja abierta o cerrada se basará en las características de los componentes y la rehabilitación que se llevará a cabo. Ambas técnicas tienen sus ventajas, sin embargo, cada una está indicada para casos específicos. En este sentido, la elección se basará en la demanda individual de cada paciente.

Palabras clave: Implantes dentales; Bandeja abierta; Bandeja cerrada; Prótesis.

\section{Introdução}

$\mathrm{Na}$ atualidade os avanços alcançados dentro da odontologia, proporcionam aos pacientes cada vez mais opções para a busca de um sorriso bonito e principalmente para a qualidade bucal. Neste sentido, buscando alcançar ambas as expectativas, as próteses sobre implantes vêm ganhando cada vez mais espaço entre as opções mais escolhidas. (Bezerra, et al., 1999).

Fernandes e seus colaboradores (2014) relatam que o aumento na procura das próteses sobre implantes se dá devido ao fato de tal técnica atingir os padrões estéticos desejados e ao mesmo tempo se consolidar como uma técnica reabilitadora que busca a preservação das condições fisiológicas do indivíduo.

No entanto, para que a técnica seja eficaz, se faz necessário além dos conhecimentos técnicos do profissional da odontologia, que este realize um bom trabalho no momento de realizar os moldes. Para tanto, a escolha da técnica de moldagem será de extrema importância para o êxito do procedimento. (Fernandes et al., 2014).

Agarwal, Ashok, e Maiti, (2020) enfatizaram que a moldagem é uma etapa importantíssima para o sucesso e longevidade de uma prótese implantossuportada, pois esta registra não só o contorno do tecido mole (perfil de emergência) e a posição hexadecimal de rotação do componente protético ou implante, mas ainda a posição (vestíbulo/lingual e mésio/distal), profundidade gengival, eixo/angulação em relação aos dentes antgônicos.

Neste sentido, o objetivo principal da moldagem, será o de reproduzir com o máximo de exatidão o posicionamento de cada componente na boca e dos tecidos moles. (Telles, 2009).

Diante do exposto o presente estudo se justifica no fato de que com o aumento da demanda por implantes dentários, se faz cada vez mais necessário que os profissionais da odontologia ampliem seus conhecimentos acerca do assunto, buscando assim, melhores resultados em seus trabalhos.

Como objetivo principal tem-se: ampliar os conhecimentos sobre as técnicas de moldagem em moldeiras abertas e fechadas. Como objetivos secundários, propõe-se: estudar sobre as próteses sobre implantes; caracterizar as distinções entre as moldeiras abertas e fechadas; ampliar os conhecimentos sobre as situações mais adequadas para o uso de cada estilo de moldeira (aberta ou fechada).

\section{Metodologia}

O presente estudo trata-se de um levantamento bibliográfico descritivo e qualitativo, utilizando-se assim de referenciais teóricos extraídos de livros, artigos científicos, monografias, teses, dissertações revistas e sites eletrônicos que retratem o tema proposto. Para tanto usou-se das bases de dados: LILACS, SCIELO, MEDLINE. As palavras-chaves utilizadas foram: prótese sobre implantes; moldeiras abertas; moldeiras fechadas; implant prosthesis; open trays; closed trays.

A revisão bibliográfica é um método utilizado em pesquisas que buscam informações mais aprofundadas e 
diversificadas de diferentes autores em determinado assunto, permitindo assim um conhecimento mais amplo do tema abordado (GERHARDT; SILVEIRA, 2009).

\section{Referencial Teórico}

\subsection{Implantes}

Nos últimos anos, a demanda para a reabilitação de perdas dentárias com implantes, se popularizou. Esse crescimento pode ser relacionado com o fato da consolidação da técnica, além da confiabilidade e redução de preço dos implantes e componentes, chegando tal diferença ao consumidor final. (Bezerra, et al., 1999).

Dentro deste contexto, é importante salientar que os implantes apresentam uma série de vantagens, como ausência de desgaste de estrutura dentária sadia, higienização mais simples e resultado estético mais satisfatório, no entanto, nem todas as situações poderão ser resolvidas por meio desta técnica. (Pimenta; Silva; Poluha, 2019).

Porém, tal técnica apresenta pontos favoráveis quando se trata de estética, fonação, retenção e estabilidade protética e na mastigação. Sendo estes, fatores significativos para o aspecto funcional e psicológico do paciente, acrescendo a autoestima e a confiança do mesmo. (Fernandes et al., 2014).

No entanto, algumas condições sistêmicas podem interferir negativamente a osseointegração, como por exemplo, os casos de pacientes com alterações hepáticas graves, diabetes e uso crônico de fumo. O fator econômico também pode interferir na escolha da técnica utilizada, visto que os implantes apresentam um custo mais elevado. (Farias Neto; Carreiro; RizzattiBarbosa, 2011).

Diante disto, e com o aumento da expectativa de vida da população e com o padrão estético atual, aqueles indivíduos portadores de prótese dental ou desdentados, passaram a desejar alternativas reabilitadoras que sejam capazes de preservar as condições fisiológicas da pessoa. Assim, as próteses sobre implantes se consolidaram como a opção mais buscada entre as alternativas viáveis para suplementação de ausência dentaria. (Fernandes et al., 2014).

Neste sentido, no atual estágio de desenvolvimento que as próteses sobre implantes atingiram, estas são consideradas como sendo as melhores opções de tratamento, sendo consideradas como de alto padrão dentro das próteses dentarias. (Rocha, et al., 2013).

No entanto, o estabelecimento da necessidade protética do paciente é realizada através de técnicas tradicionais de planejamento que abrangem a utilização de anamnese do paciente, exames intra-orais e extra orais, montagem de modelos de estudo em articulador semi-ajustáveis e procedimentos diagnósticos complementares como radiografias, tomografias e prototipagem (caso seja necessária). (Telles, 2009).

É importante destacar que o motivo do sucesso no tratamento reabilitador com prótese sobre implantes, é que além do paciente ficar satisfeito, este consegue percebe de maneira rápida que a falta de um ou mais dentes vai além das questões estéticas, abrangendo também problemas funcionais como dificuldades na mastigação e na fonética. (Fernandes et al., 2014).

A instalação de implantes é limitada inicialmente a algumas situações como: proximidade do canal mandibular, redução da altura óssea, extensa pneumatização e acidentes anatômicos. Assim, nos casos em que ocorrem essas limitações anatômicas e/ou fisiológicas, várias técnicas de enxertia óssea podem ser propostas. Além disso o aprimoramento também evoluiu quanto ao tamanho, largura, geometria interna (conexões e junções) e externa (formato e espiras), tratamento de superfície e material de confecção dos implantes. (Santiago et. al., 2010).

O tipo de interface protética, a presença ou ausência de roscas, macro irregularidades adicionais e o formato externo do corpo do implante constituem aspectos importantes do seu desenho. (Santiago et al., 2010).

Os materiais que atualmente são utilizados em próteses sobre implantes são aqueles chamados de biocompatíveis que apresentam propriedades mecânicas e de corrosão adequada para sua aplicação. Entre os materiais utilizados estão: titânio e suas 
ligas, aço inoxidável, ligas de níquel-crômio, cromo-cobalto, tântalo, ouro-paládio e prata-paládio. Existe ainda os materiais considerados metal free, onde os componentes e prótese são confeccionados de cerâmicas, sendo a zircônia a mais conhecida e empregada. Tal condição também é possível para a confecção dos implantes, onde já existe no mercado, implantes e fresas livres de metal confeccionados de zircônia. (Bezerra, et al., 1999).

Além das questões relacionadas com biocompatibilidade, uma prótese sobre implantes deverá apresentar biofuncionalidade. Em outras palavras, esta deverá possuir capacidade para desempenhar apropriadamente a função para a qual a mesma é desenvolvida. (Bezerra, et. al., 1999).

Neste sentido, o sucesso do tratamento com implantes estará relacionado de maneira direta com a elaboração de um plano de tratamento correto, independente de ser a reposição de um único dente ou de toda arcada dentária. Assim, antes da instalação dos implantes, o paciente recebe o preparo e planejamento protético prévio, onde o protesista terá uma noção de qual tipo de prótese será utilizada, definindo se a mesma será cimentada ou parafusada, bem como o número e distribuição de implantes adequada para a reabilitação. (Mendes; Rohenkohl; Mendes, 2010).

\subsection{Moldeira fechada e moldeira aberta}

Para que uma reabilitação oral seja considerada eficaz, é de suma importância que se consiga reproduzir em laboratório, de maneira satisfatória, as estruturas que constituem a base de suporte protético na boca, seja estes dentes ou implantes. Neste sentido, o procedimento de moldagem deve ser muito bem realizado, para que os modelos de trabalho obtidos realmente retratem as condições bucais, com distorção mínima, para que todas as etapas seguintes sejam realizadas de forma efetiva. (Telles, 2009).

Assim, uma moldagem inadequada pode acabar por resultar em desajuste da prótese e como consequência, ocasionar complicações mecânicas como afrouxamento do parafuso, fratura do implante, má-oclusão, fratura do parafuso entre outras, e/ou complicações biológicas como reações inflamatórias nos tecidos biológicos entre outros. (Marotti; Tortamano; Wolfart, 2012).

A moldagem dos implantes é uma etapa de fundamental importância em um tratamento reabilitador, uma vez que os modelos obtidos devem representar precisamente o relacionamento intrabucal dos implantes. Dessa forma, é possível a obtenção de próteses com ajuste passivo, sem a ocorrência de tensões nos componentes do implante, na interface ossoimplante e no tecido ósseo circunvizinho. Idealmente, uma técnica de moldagem deveria ser realizada num menor tempo possível, ser de fácil execução, de baixo custo, confortável para o paciente e permitir a obtenção de modelos precisos. (Silva, et. al., 2008, p. 306).

Partido deste pressuposto, o principal objetivo de uma moldagem em prótese sobre implantes é registrar com exatidão o posicionamento dos componentes na boca e dos tecidos moles, bem como das estruturas adjacentes ao espaço edêntulo, utilizando-se assim componentes específicos para transferência, bem como análogos metálicos do dispositivo presente em boca, seja pilares ou implantes. (Telles, 2009).

Quando se fala em técnicas com moldeira aberta ou fechada, é importante destacar que as ferramentas utilizadas em cada uma devem ser específicas para aquele fim. Nesse sentido, quando falamos de prótese sobre implantes alguns dispositivos são utilizados para que o mesmo obtenha sucesso. No processo de moldagem, podemos destacar os transferentes ou transfers, que são dispositivos que são adaptados na plataforma do implante ou do pilar protético, transferindo a partir de uma técnica de moldagem, a posição e o formato desses objetos para o modelo de trabalho. Alguns pilares não necessitam de transfer, como alguns apresentam um tipo de transfer, e outros apresentam os 2 tipos de transferes: para moldeira aberta e fechada. (Kono, 2014).

\subsubsection{Moldeira aberta}

O estilo de moldagem com moldeira aberta é uma técnica onde se utiliza de moldeiras e componentes de moldagens que são elaborados especialmente para esse fim e com isso permitem transferir com grande exatidão a posição tanto da plataforma 
e/ou da cabeça de um implante, quanto de algum componente intermediário protético. (Telles, 2009).

Essa técnica de moldagem é utilizada quando é necessário um maior grau de precisão, em especial nos casos de implantes adjacentes, divergentes e também em pacientes desdentados, bem como em casos unitários. Após parafusar os transferentes nos implantes através de parafusos-guia, uma moldeira plástica aberta na região oclusal, individual ou de estoque deve ser utilizada. (Marotti; Tortamano; Wolfart, 2012).

É importante destacar que as moldeiras abertas podem ser compradas para este fim, onde são confeccionadas de material plástico ou acrílico e apresentam janelas destacáveis na oclusal que são removidas apenas no local onde será utilizado o transfer, ou ainda pode-se utilizar moldeiras convencionais de plástico ou acrílico que devem ser perfuradas também conforme necessidade, na posição dos transferentes. (Marotti; Tortamano; Wolfart, 2012; Kono, 2014).

Esta moldagem é a mais confiável, pois garantem o posicionamento exato do implante ou pilar, tanto da altura (cérvicooclusal), quanto ainda da posição do antirotacional uma vez que o transferente é removido junto no molde, durante o processo de moldagem. (Vargas, 2017).

\subsubsection{Moldeira fechada}

A técnica de moldagem com moldeira fechada é considerada como sendo uma técnica mais simples e que possibilita a transferência da posição da cabeça de um implante ou de algum componente protético intermediário. Em outras palavras, consiste em conectar um componente apropriado para moldagem ao implante ou mesmo ao pilar intermediário e assim executar um procedimento de moldagem do arco, reproduzindo assim, a forma dos dentes e componentes e com isso, realizando o registro da posição destes em relação ao conjunto. (Telles, 2009).

Esta técnica é usualmente utilizada nos casos em que serão realizados implantes unitários ou de até três elementos que não sejam adjacentes entre si, usualmente em pacientes dentados. Estes transfer ficam agarrados na boca durante a moldagem e remoção do molde. Depois da obtenção do molde, cada transferente será removido da boca e parafusado no seu análogo, e em seguida será encaixado manualmente no molde buscando-se assim, visualmente, a posição exata de como estava na boca. (Marotti; Tortamano; Wolfart, 2012).

Dentro deste contexto, os transferentes cônicos que são utilizados nas moldeiras fechadas, possibilitam que o análogo seja parafusado fora do molde, possibilitando uma melhor visualização da adaptação entre os componentes, no entanto, o reposicionamento dos transferentes no molde, pode possibilitar uma alteração de posição dentro do material de moldagem antes do vazamento, tanto do posicionamento do antirotacional, quanto ainda do posicionamento espacial no sentido cérvico-oclusal. (Vargas, 2017).

Neste estilo de moldagem, utiliza-se de moldeiras convencionais metálicas ou plásticas. (KONO, 2014).

Existem pilares protéticos mais atuais que apresentam apenas um tipo de transfer, o de moldeira fechada. Tais componentes foram desenvolvidos buscando agilizar o processo de moldagem, mas evitar problemas referentes à falha no posicionamento tridimensional do componente na cavidade bucal. Desta forma, estes componentes foram desenvolvidos com retenções em sua superfície externa, e ainda com clips de retenção em vez de parafusos, para que durante a remoção da moldeira da boca, estes destaquem juntamente à moldagem, oferecendo um molde similar ao de moldeira aberta. (SOUZA, et al., 2021).

\section{Discussão}

Os autores são unânimes ao relatarem que nos últimos anos, a demanda para a reabilitação dentária vem crescendo de maneira exponencial. Este fato pode estar relacionado com a popularização da técnica.

Fernandes e colaboradores (2014) destacam como pontos positivos dos implantes dentários seus resultados na estética, na retenção e estabilidade protética, além da melhora na fonação e mastigação do paciente. 
Em contra partida, outros autores como Farias Neto, Carreiro e Rizzatti-Barbosa (2011) e Santiago e colaboradores (2010) não desconsideram as vantagens observadas por Fernandes e colaboradores (2014), porém destacam a existência de condições que podem interferir de maneira negativa na implantação dentária, como por exemplo, pacientes com alterações hepáticas graves, diabetes e uso crônico de fumo.

Além disso, destacam que alguns fatores como proximidade do canal mandibular, redução da altura óssea, extensa pneumatização e acidentes anatômicos, podem ser limitantes para o uso da técnica.

Um ponto importante que foi destacado pelos autores Telles (2009); Marotti, Tortamano e Wolfart (2012); Silva e colaboradores (2008); e kono (2014) foi à importância de que o processo inicial de moldagem seja realizado de maneira eficaz, uma vez que uma moldagem inadequada contribui para que ocorra um desajuste da prótese, podendo inclusive ocasionar complicações mecânicas. Neste sentido, a escolha do tipo de moldeira a ser utilizada (aberta ou fechada) é um passo importante em todo o processo.

Em relação a moldeira aberta Vargas (2017), salienta que se trata de um tipo de moldagem mais confiável, devido ao fato de que com essa moldeira o posicionamento do implante ou do pilar será mais exato tanto em relação a altura como também em relação a posição antirotacional.

Em conformidade com a posição de Vargas (2017), Marotti, Tortamano e Wolfart (2012) bem como Talles (2009) sugerem o uso da moldeira aberta para aqueles casos onde se faz necessário um maior nível de precisão, como nos casos de implantes adjacentes, divergentes ou em casos unitários ou em pacientes desdentados. No entanto, Amin e colaboradores (2017) mostra que as técnicas de escaneamento podem ser mais precisas que a técnica de moldeira aberta convencional. E em contrapartida Kim, Seo e Kim (2019) encontraram valores mais precisos para a técnica convencional quando moldagem de implantes em arcada desdentada total.

Estes mesmos autores, compartilham da premissa de que a técnica de moldagem com moldeiras fechadas é uma técnica mais simples, sendo utilizada em casos de implantes unitários ou de até 03 elementos, desde que estes não estejam adjacentes entre si, além de ser utilizada em pacientes dentados.

Vale ressaltar que o encaixe de transfers no momento da moldagem deve ser conferido, principalmente em casos unitários, sendo muito importante tal análise clínica e até radiográfica, principalmente nos casos onde estes componentes são encaixados muito subgengivalmente.

Novas tecnologias vem sendo desenvolvidas e criadas para facilitar não só a captura dos componentes/implantes no espaço 3D da arcada dentária, como ainda para a confecção das próteses dentárias (Amin et al., 2017; Liu et al., 2019; Wolfart \& Yilmaz, 2019).

\section{Considerações Finais}

O contexto atual exige cada vez mais naturalidade de um sorriso, com isso vários avanços foram alcançados dentro da odontologia, em especial dentro da Implantodontia. Nesse sentido, as próteses sobre implantes estão sendo cada vez mais procuradas.

As vantagens de utilizar-se desta técnica vão desde maior naturalidade no sorriso até o fato de que tal procedimento possui melhores resultados em longo prazo. Assim, conclui-se que para que a prótese sobre implantes tenha melhor êxito, é fundamental que todos os passos de sua implantação sejam executados com sucesso, incluindo aqui o momento da moldagem.

Portanto, a escolha entre moldeira aberta ou fechada, será baseada nas características dos componentes e reabilitação que serão realizados. Ambas as técnicas possuem suas vantagens, no entanto, cada uma é indicada para casos específicos. Neste sentido, a escolha se baseará na demanda individual de cada paciente. 
Research, Society and Development, v. 10, n. 10, e265101018749, 2021

(CC BY 4.0) | ISSN 2525-3409 | DOI: http://dx.doi.org/10.33448/rsd-v10i10.18749

\section{Referências}

Agarwal, S., Ashok, V., \& Maiti, S. (2020). Open- or Closed-Tray Impression Technique in Implant Prosthesis: A Dentist's Perspective. Journal of long-term effects of medical implants, 30(3), 193-198. https://doi.org/10.1615/JLongTermEffMedImplants.2020035933

Amin, S., Weber, H. P., Finkelman, M., El Rafie, K., Kudara, Y., \& Papaspyridakos, P. (2017). Digital vs. conventional full-arch implant impressions: a comparative study. Clinical oral implants research, 28(11), 1360-1367. https://doi.org/10.1111/clr.12994

Bezerra, R. M. et al. (1999). Microestrutura e resistência à corrosão do Ti c.p. soldado a laser utilizando em prótese sobre implantes. Elétrica Química, 24, 113124.

Farias Neto, A., Carreiro, A. F. P. \& Rizzatti-Barbosa, C. M. A. (2011). Prótese parcial removível no contexto da odontologia atual. Odontologia ClínicoCientífica,10 (2), 125-8.

Fernandes JR., R. C. et al. (2014). Implantodontia: próteses totais fixas sobre implante com carga imediata em mandíbula. Revista de Iniciação Científica da Universidade Vale do Rio Verde, 4 (1), 76-93.

Gerhardt, T. E. \& Silveira, D. T. (2009). Métodos de pesquisa. Editora da UFRGS.

Kim, K. R., Seo, K. Y., \& Kim, S. (2019). Conventional open-tray impression versus intraoral digital scan for implant-level complete-arch impression. The Journal of prosthetic dentistry, 122(6), 543-549. https://doi.org/10.1016/j.prosdent.2018.10.018

Kono, T. T. L. D. V. (2014). Especialização em Odontologia (Monografia). Universidade Estadual de Campinas.

Liu, D. Y., Cader, F. N., Abduo, J., \& Palamara, J. (2019). Accuracy of Different Implant Impression Techniques: Evaluation of New Tray Design Concept. Journal of prosthodontics: official journal of the American College of Prosthodontists, 28(2), e682-e687. https://doi.org/10.1111/jopr.12733

Marotti, J., Tortamano, P. \& Wolfart, S. (2012). Moldagem em implantodontia. Revista de Pós-Graduação, 19 (3), $113-21$.

Mendes, L. G. A., Rohenkohl, J.H. \& Mendes, M. O. A. (2010). Prótese sobre implantes: cimentada versus parafusada. Unoesc \& Ciência, 1 (2), $157-164$.

Pimenta, M. H. G., Silva, R. S. S. \& Poluha, R. L. (2019). Moldagem Em Prótese Sobre Implante: Revisão De Literatura. Revista UNINGÁ, 56 (S5), $37-42$.

Rocha, S. S. et. al. (2013). Próteses Totais Fixas Tipo Protocolo Bimaxilares. Relato de Caso. Revista Odontologia do Brasil Central, 21 (60), $21-7$.

Santiago J. R. et al. (2010). Implantes dentais curtos: alternativa conservadora na reabilitação bucal. Revista de Cirurgia e Traumatologia Buco-maxilo-facial, $10(2), 67-76$

Silva, M. M. et. al. (2008). Técnicas de moldagem em prótese sobre implantes. Revista de Odontologia da UNESP, 37 (4), $301-8$.

Souza, S. C. et al. (2021). Análise comparativa entre diferentes técnicas de união de transferentes de moldagem utilizadas em prótese sobre implantes cone morse. Arch Health Invest, 10 (3), $461-6$.

Telles, D. (2009). Prótese total: convencional e sobre implantes. Santos.

Vargas, F. F. (2017). Especialização em Odontologia (Dissertação). Universidade Federal de Pelotas.

Wolfart, S., \& Yilmaz, B. (2019). A technique for facilitating open-tray implant impressions. The Journal of prosthetic dentistry, 122(4), 417-419. https://doi.org/10.1016/j.prosdent.2019.06.017 